\title{
Anomalous dimensions from rotating open strings in AdS/CFT
}

\author{
Nelson R.F. Braga ${ }^{a}$ and Edmond lancu ${ }^{b}$ \\ ${ }^{a}$ Instituto de Física, Universidade Federal do Rio de Janeiro, \\ Caixa Postal 68528, RJ 21941-972, Brazil \\ ${ }^{b}$ Institut de Physique Théorique de Saclay, \\ F-91191 Gif-sur-Yvette, France \\ E-mail: braga@if.ufrj.br, edmond.iancu@cea.fr
}

ABSTRACT: We propose a new entry within the dictionary of the AdS/CFT duality at strong coupling: in the limit of a large spin or a large R-charge, the anomalous dimension of the gauge theory operator dual to a semiclassical rotating string is proportional to the string proper length. This conjecture is motivated by a generalization to strings of the rule for computing anomalous dimensions of massive particles and supergravity fields in the anti-de Sitter space. We show that this proportionality holds for a rotating closed string in global AdS space, representing a high spin operator made of fields in the adjoint representation. It is also valid for closed strings rotating in $S^{5}$ (representing operators with large R-charge), for closed strings with multiple AdS spin, and for giant magnons. Based on this conjecture, we calculate the anomalous dimension $\delta$ of operators made of fields in the fundamental representation, associated with high spin mesons, and which are represented by rotating open strings attached to probe D7-branes. The result is a logarithmic dependence upon the spin, $\delta \sim \sqrt{\lambda} \ln S$, similar to the closed string case. We show that the operator properties - anomalous dimension and spin - are obtained from measurements made by a local observer in the anti-de Sitter space. For the open string case, this ensures that these quantities are independent of the mass scale introduced by the D7-branes (the quark mass), as expected on physical grounds. In contrast, properties of the gauge theory states, like the energy, correspond to measurements by a gauge theory observer and depend upon the mass scale - once again, as expected.

KeYwords: AdS-CFT Correspondence, Gauge-gravity correspondence

ARXIV EPRINT: 1405.7388 


\section{Contents}

1 Introduction 1

2 From the string length to anomalous dimensions $\quad 4$

3 Testing our conjecture versus closed strings $\quad 6$

$\begin{array}{ll}3.1 \text { Closed string with high spin } & 7\end{array}$

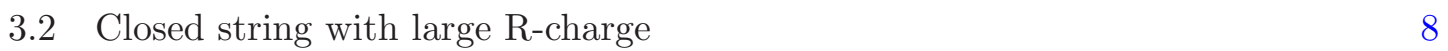

3.3 Multi-spin closed string $\quad 9$

$\begin{array}{lr}3.4 \text { Giant magnon } & 10\end{array}$

4 Open strings and high spin mesons 10

$\begin{array}{llr}5 & \text { Final comments } & 14\end{array}$

\section{Introduction}

Anomalous dimensions of high spin operators are important ingredients of gauge theories. In perturbative QCD, the anomalous dimension grows with the logarithm of the spin. This property played an essential role in the analysis of the approach to Bjorken scaling in deep inelastic scattering, for $\left|q^{2}\right| \rightarrow \infty$, as a consequence of asymptotic freedom $[1,2]$. The logarithm dependence for high spins appears to be valid at all loop orders [3-5].

At strong coupling, it is possible to investigate gauge theories using the gauge/string duality [6-8]: for the case of $\mathcal{N}=4$ supersymmetric Yang Mills (SYM) theory, the AdS/CFT correspondence provides a 'dual' description in terms of a string theory living in the $\mathrm{AdS}_{5} \times \mathrm{S}^{5}$ space-time. This in particular allows one to compute the anomalous dimensions of high-spin operators at strong coupling [9]. Within the context of the pure-gauge $\mathcal{N}=4$ SYM theory, such operators are exclusively built with fields in the adjoint representation of the gauge group $\mathrm{SU}(N)$. Specifically, ref. [9] computed the anomalous dimensions of high-spin, twist-two, operators like $\operatorname{Tr}\left(\phi^{I} D_{\left(\mu_{1} \ldots\right.} D_{\left.\mu_{S}\right)} \phi^{I}\right)$ via studies of the corresponding 'dual' object — a semiclassical closed string rotating in global $\mathrm{AdS}_{5}$ space-time. Within this duality, time translations in global $\mathrm{AdS}_{5}$ correspond to dilatations in the gauge theory. Accordingly, the conformal dimension $\Delta$ of the gauge-theory operator can be identified with the energy of the rotating string. In the high-spin limit $S \gg \sqrt{\lambda}$, this method yields a logarithmic dependence of the anomalous dimension $\delta=\Delta-S$ upon the spin $S$, which is similar to that found in perturbation theory (in both QCD and $\mathcal{N}=4 \mathrm{SYM}$ ):

$$
\delta=\Delta-S \simeq \frac{\sqrt{\lambda}}{\pi} \ln \left(\frac{S}{\sqrt{\lambda}}\right)
$$

where $\lambda=g_{\mathrm{YM}}^{2} N$ is the 't Hooft coupling and $g_{\mathrm{YM}}$ is the coupling of the gauge theory. 
Within perturbative QCD, a similar behavior at large $S$ holds also for the twist-two operators built with quark fields in the fundamental representation of $\mathrm{SU}(N)$, with the general structure

$$
\mathcal{O}_{S}=\bar{\Psi} \gamma^{\left(\mu_{1}\right.} D^{\mu_{2}} \ldots D^{\left.\mu_{S}\right)} \Psi
$$

where $\Psi$ denotes the quark field. Once again, one expects this logarithmic behavior $\delta \propto \ln S$ for $S \rightarrow \infty$ to hold to all orders in perturbation theory, and also at strong coupling (at least, within gauge theories involving matter fields in the fundamental representation and for which the strong coupling limit is clear to make sense, like the $\mathcal{N}=2$ Yang Mills theory that we shall focus on in this work). Besides explicit calculations in perturbation theory at low orders, there are solid non-perturbative arguments in that sense.

One such an argument comes from the relation between the anomalous dimension of twist-two operators with high spin $S$ and the anomalous dimension of a cusp in a light-like Wilson line [3, 4]: the coefficient $f(\lambda)$ in the asymptotic expression $\delta \simeq f(\lambda) \ln S$ for $\delta$ at large $S$ coincides with the cusp anomalous dimension. This relation is conjectured to hold regardless of the value of the coupling and for any gauge-group representation of the matter fields within the structure of the operator. (This has been checked in perturbation theory in many gauge theories, including non-supersymmetric ones, and also at strong coupling in the context of $\mathcal{N}=4$ SYM [10-14].) If extrapolated at strong coupling, it predicts that the anomalous dimension of the quark-antiquark operator in eq. (1.2) at large $S$ should be half $^{1}$ of the corresponding result for an adjoint operator, as shown in eq. (1.1).

Another compelling argument comes from the geometrical interpretation, proposed in [15], for the $\ln S$-dependence of the anomalous dimension $\delta$ at large $S$, and also for its relation to the cusp anomaly. Once again, this interpretation is expected to hold for any value of the coupling and irrespective of the gauge-group representation.

Notwithstanding, it would be interesting to have a direct calculation at strong coupling of the anomalous dimensions associated with high-spin operators involving fundamental matter fields, cf. eq. (1.2). In this paper, we shall propose a method in that sense, based on the study of rotating open strings in the $\mathrm{AdS}_{5} \times \mathrm{S}^{5}$ space-time represented in Poincaré coordinates.

To describe our method, let us first recall that, in order to include fields in the fundamental representation of $\mathrm{SU}(N)$ ('quarks') in AdS/CFT, one must introduce probe D7branes in $\mathrm{AdS}_{5}$ [16]. The dual gauge theory is the $\mathcal{N}=2$ Yang Mills theory with massive quarks. Mesons of low spin are described as fluctuations of the D7-branes while high spin mesons are represented by semiclassical rotating open strings attached to the D7-branes in a Poincaré $\mathrm{AdS}_{5}$ space [17] (see also [18] for a review). The presence of the D7-branes introduces a mass scale in the duality, the quark mass $M_{q}$, corresponding to the position of the branes along the 'radial' direction of $\mathrm{AdS}_{5}$. As a consequence, a rotating open string representing a high spin meson is not invariant under dilatations. This situation contrasts with the closed string case, where the dual gauge theory is conformal. So, the approach pioneered in [9], which consists in computing the anomalous dimension $\delta$ from the dilata-

\footnotetext{
${ }^{1}$ Indeed, within the planar limit $N \rightarrow \infty$ under consideration, a Wilson loop in the adjoint representation is equal to the square of the same loop in the fundamental representation; hence, $f_{\text {adj }}(\lambda)=2 f_{\text {fdt }}(\lambda)$.
} 
tion charge $\Delta=S+\delta$ (which in turn is dual to the energy of a rotating closed string in global $\mathrm{AdS}_{5}$ ), cannot be carried over to the case of an open string.

As an alternative approach, valid to parametric accuracy in the limit of a large spin and/or large R-charge, we propose a method to compute the anomalous dimension $\delta$ of the gauge theory operator dual to a semiclassical (closed or open) string from the string proper length $\ell$ in the temporal gauge. More precisely, introducing the dimensionless 'scale parameter' $\gamma \equiv R T_{0} \ell$, where $R$ is the $\mathrm{AdS}_{5}$ radius and $T_{0}=1 /\left(2 \pi \alpha^{\prime}\right)$ is the string tension, we conjecture that $\gamma$ and $\delta$ are proportional to each other in the semiclassical limit alluded to above. The proportionality constant (a pure number) is however not uniquely determined, as we shall see on specific examples.

Our proposal, to be detailed in section 2, is motivated by the analogy with the corresponding calculations for (very) massive supergravity objects which are localized in $\mathrm{AdS}_{5}$, like supergravity fields [7, 8] and point-like AdS particles [19]. In these well-known examples, the anomalous dimensions of the respective dual operators in the semiclassical limit $m R \gg 1$ are obtained as $\delta \simeq m R$, with $m$ the mass of the supergravity dual. As we shall explain in section 2, our scale parameter $\gamma$ is proportional to the integral over the string of the masses of infinitesimal string bits measured by local observers in AdS (so, in that sense, it plays the same role as the mass of an AdS particle). Then, in section 3, we shall test our conjecture on several examples involving closed strings (dual to operators made of adjoint fields), for which the respective anomalous dimensions have already been computed, via the recipe introduced in ref. [9]. We shall thus demonstrate that the string length is indeed proportional to the known anomalous dimensions, in several cases: a closed string with a large spin $S$ [9], one with a large R-charge [9], one with large multiple-spin [20], and finally a giant magnon [21] .

In section 4, we address the main physics problem which has motivated our analysis: the calculation of the anomalous dimension for the quark-antiquark twist-two operator in eq. (1.2) at large $S$ and strong coupling. To that aim, we shall assume that the string-theory object dual to this operator is an open string rotating in $\operatorname{AdS}_{5}$, with the string endpoints attached to a D7-brane. This identification is by no means obvious (in fact, we are not aware of similar observations in the literature), so let us briefly explain our motivation for it. The main argument in that sense is an a posteriori one, namely, the consistency between this (conjectured) duality and our final result for $\gamma$ (the scale parameter for a rotating open string). Specifically, in the high-spin limit $S \gg \sqrt{\lambda}$, we shall find $\gamma \simeq 2(\sqrt{\lambda} / \pi) \ln (S / \sqrt{\lambda})$, which is the expected result for the anomalous dimension of the quark operator in eq. (1.2), to parametric accuracy. The numerical prefactor is probably wrong: as compared to the respective result for an adjoint operator, cf. eq. (1.1), we have a factor of 2 , instead of the expected $1 / 2$. But as already mentioned, this numerical coefficient is not under control within our method, and should not be trusted.

Another strong argument in favor of the duality between the rotating open string and the quark-antiquark operator (1.2) is the fact that the respective scale parameter $\gamma$ is found to be independent of the infrared scale (the quark mass $M_{q}$ ) of the gauge theory, as expected for the anomalous dimension of a local operator. This scale invariance, which is manifest on the expression for $\gamma$ in terms of $S$ shown above, emerges from the string calculation in 
a very interesting way: the proper length $\ell$ of the open string remains invariant under the $\mathrm{AdS}_{5}$ isometry corresponding to dilatations in the gauge theory. The effect of this isometry is to rescale the radial position of the D7-brane, which in turn corresponds to a redefinition of the quark mass $M_{q}$ in the gauge theory. This invariance is quite non-trivial ${ }^{2}$ - it does not hold e.g. for the energy of the string (which scales with the quark mass like $M_{q}$ ), nor for the separation between the string endpoints on the D7-brane (which scales like $1 / M_{q}$ and is indicative of the physical size of the meson state in the dual gauge theory). It is related to the fact that, as alluded to above, the scale parameter $\gamma$ is obtained from string properties as measured by a local observer in the AdS space-time. That is, this invariance is tributary to the warp factor relating local densities on the string to the corresponding densities measured by a gauge-theory observer.

This discussion sheds new light on the way how the AdS/CFT correspondence works in the presence of mass scales, as introduced by D7-branes. A local measurement in AdS yields results which are independent of the mass scale and hence can be attributed to a local operator. A gauge-theory observer, on the other hand, measures properties of the gauge-theory state, like its energy or average size, which are sensible to the mass scale $M_{q}$. The case of the spin is particularly interesting in that respect: this is at the same time an attribute of the operator, cf. eq. (1.2), and of the meson state created by this operator. And indeed, as we shall further discuss in section 4, both local observers in $\mathrm{AdS}_{5}$, and boundary observers using the gauge-theory time, find the same value $S$ for the angular momentum of the string.

\section{From the string length to anomalous dimensions}

We consider a classical string embedded in the target space-time $\mathrm{AdS}_{5} \times \mathrm{S}^{5}$ with metric $g_{m n}$. For definiteness, in this section we consider $\mathrm{AdS}_{5}$ with Minkowskian signature and Poincaré coordinates, but our discussion below would also apply to an Euclidean space-time, and also to $\mathrm{AdS}_{5}$ with global metric. A convenient choice of coordinates is

$$
\mathrm{d} s^{2}=\frac{R^{2}}{z^{2}}\left(-\mathrm{d} t^{2}+\mathrm{d} \boldsymbol{x}^{2}+\mathrm{d} z^{2}\right)+R^{2} \mathrm{~d} \Omega_{5}^{2} \equiv g_{m n} \mathrm{~d} x^{m} \mathrm{~d} x^{n},
$$

where $z$ is the holographic radial coordinate, $x^{\mu}=\left(x^{0}, x^{1}, x^{2}, x^{3}\right) \equiv(t, \boldsymbol{x})$ are the coordinates in the Minkowski space at the boundary of $\mathrm{AdS}_{5}$ at $z=0$, and $\mathrm{d} \Omega_{5}^{2}$ refers to $\mathrm{S}^{5}$ (see eq. (3.12) below for an explicit map on $\mathrm{S}^{5}$ ). The string embedding is parametrized as $X^{m}=X^{m}(\tau, \sigma)$, where $\tau$ and $\sigma$ are coordinates on the string world-sheet. The dynamics of the string is governed by the Nambu-Goto action

$$
I=-\frac{1}{2 \pi \alpha^{\prime}} \int \mathrm{d} \tau \mathrm{d} \sigma \sqrt{-\operatorname{det} h_{\alpha \beta}}, \quad h_{\alpha \beta}=g_{m n} \partial_{\alpha} X^{m} \partial_{\beta} X^{n},
$$

where $\alpha, \beta=0$ or $1,\left(\sigma^{0}, \sigma^{1}\right) \equiv(\tau, \sigma)$, and $h_{\alpha \beta}$ is the induced world-sheet metric. Writing $h \equiv \operatorname{det} h_{\alpha \beta}$, one has

$$
-h=\left(\dot{X} \cdot X^{\prime}\right)^{2}-(\dot{X})^{2}\left(X^{\prime}\right)^{2}
$$

\footnotetext{
${ }^{2}$ This invariance has also been exploited in ref. [17], at least implicitly (via a judicious choice of coordinates), but its consequences - like the scale invariance of the string calculation for the spin — have not been discussed there.
} 
where a dot (prime) refers to the derivative w.r.t. $\tau(\sigma), A \cdot B \equiv g_{m n} A^{m} B^{n}$, and $A^{2} \equiv A \cdot A$.

We chose the temporal gauge $\tau=X^{0}$ and define

$$
\gamma \equiv \frac{R}{2 \pi \alpha^{\prime}} \ell \equiv \frac{R}{2 \pi \alpha^{\prime}} \int_{\text {string }} \mathrm{d} \sigma \sqrt{g_{m n} \frac{\partial X^{m}}{\partial \sigma} \frac{\partial X^{n}}{\partial \sigma}}
$$

For definiteness, the quantities $\gamma$ and $\ell$ will be referred to as the 'string scale parameter' and, respectively, the 'string proper length'. For what follows, it is useful to notice that the above integral can be equivalently rewritten as

$$
\gamma=R \int_{\text {string }} \mathrm{d} \sigma \sqrt{-g^{m n} \pi_{m}^{0} \pi_{n}^{0}}
$$

where $\pi_{m}^{0}$ is the density of the $m$-component of the canonical momentum on the string world-sheet:

$$
\pi_{m}^{0} \equiv \frac{\partial \mathcal{L}}{\partial \dot{X}^{m}}=-T_{0} g_{m n} \frac{\left(\dot{X} \cdot X^{\prime}\right)\left(X^{n}\right)^{\prime}-\left(X^{\prime}\right)^{2}\left(\dot{X}^{n}\right)}{\sqrt{-h}}
$$

with $\mathcal{L}$ the Lagrangian density in eq. $(2.2)$ and $T_{0}=1 / 2 \pi \alpha^{\prime}$ the string tension. Indeed, by using eqs. (2.3) and (2.6), one can easily check the identity $\sqrt{-g^{m n} \pi_{m}^{0} \pi_{n}^{0}}=\sqrt{\left(X^{\prime}\right)^{2}}$.

For a string living in a flat space-time with Minkowski metric $\eta^{m n}$, the equivalence between the integral expressions in eqs. (2.4) and respectively (2.5) has a limpid physical interpretation. The quantity $\mathrm{d} m \equiv T_{0} \mathrm{~d} \ell$ represents the mass of an infinitesimal piece of string with length $\mathrm{d} \ell=\sqrt{\left(X^{\prime}\right)^{2}} \mathrm{~d} \sigma$. Similarly, $\mathrm{d} P_{m} \equiv \pi_{m}^{0} \mathrm{~d} \sigma$ is the (infinitesimal) spacetime momentum of that piece of string. Hence the relation $\mathrm{d} m=\sqrt{-\eta^{m n} \mathrm{~d} P_{m} \mathrm{~d} P_{n}}$ is recognized as the mass-shell condition for a relativistic 'particle' (here, a bit of the string). The overall space-time momentum of the string is computed by integrating the momentum density over the string length: $P_{m}=\int \mathrm{d} \sigma \pi_{m}^{0}$. The mass $M$ of the string is finally obtained as $M=\sqrt{-\eta^{m n} P_{m} P_{n}}$.

But for a string living in the curved AdS space-time, the situation is more subtle because the space-time momenta look different to an inertial observer living in the gauge theory ('on the Minkowski boundary of $\mathrm{AdS}_{5}$ ') as opposed to a local observer in ten dimensions. This difference is due to the gravitational redshift (warp factor) $\sqrt{g_{00}}=R / z$ multiplying the flat boundary metric in eq. (2.1). Yet, as we now argue, it is natural to interpret the integrand in eq. (2.5) as the local 'mass' (or 'virtuality') of a bit of the string, as viewed by a local observer in AdS space (i.e. an observer whose instantaneous position in ten dimensions is the same as that of a point on the string and whose local time is $\left.\bar{t} \equiv \sqrt{g_{00}} t=(R / z) t\right)$. To see this, consider for simplicity a string stretching and moving only in $\mathrm{AdS}_{5}$, which has no momenta along the $\mathrm{S}^{5}$ directions; for such a string, one has (with sum over $i=1,2,3$ )

$$
\mathrm{d} \sigma \sqrt{-g^{m n} \pi_{m}^{0} \pi_{n}^{0}}=\mathrm{d} \sigma \frac{z}{R} \sqrt{\left(\pi_{0}^{0}\right)^{2}-\left(\pi_{i}^{0}\right)^{2}-\left(\pi_{z}^{0}\right)^{2}}=\sqrt{(\mathrm{d} \bar{E})^{2}-\left(\mathrm{d} \bar{P}^{i}\right)^{2}-\left(\mathrm{d} \bar{P}^{z}\right)^{2}},
$$

where

$$
\frac{\mathrm{d} \bar{E}}{\mathrm{~d} \sigma}=-\frac{z}{R} \pi_{0}^{0}, \quad \frac{\mathrm{d} \bar{P}^{i}}{\mathrm{~d} \sigma}=\frac{z}{R} \pi_{i}^{0}, \quad \frac{\mathrm{d} \bar{P}^{z}}{\mathrm{~d} \sigma}=\frac{z}{R} \pi_{z}^{0},
$$


are the string densities of the respective canonical momenta, as seen by the local observer. They differ via the warp factor $z / R$ from the respective densities which enter the calculation of the physical 4-momentum, as seen by a gauge theory observer:

$$
E=-\int \mathrm{d} \sigma \pi_{0}^{0}, \quad P^{i}=\int \mathrm{d} \sigma \pi_{i}^{0} .
$$

This difference is important for our present purposes, in that it shows that the quantity $\mathrm{d} \bar{m} \equiv \mathrm{d} \sigma \sqrt{-g^{m n} \pi_{m}^{0} \pi_{n}^{0}}$ can be viewed as the mass of a bit of string in AdS, which in turn suggests an interesting physical interpretation for $\gamma=R \int_{\text {string }} \mathrm{d} \bar{m}$.

Namely, it is well known that the AdS masses of local objects in $\mathrm{AdS}_{5}$, like supergravity fields or massive point-like particles, are proportional to the anomalous dimensions of the corresponding operators in the dual gauge theory $[7,8]$. The example of a point particle in $\mathrm{AdS}_{5}$ with mass $m$ is particularly inspiring for our present purposes. In the semiclassical limit where $m R \gg 1$, the anomalous dimension of the CFT operator dual to this AdS particle is found as $\delta \simeq m R$ (see [19] for an explicit calculation). Note that this mass $m$ is a property measured by a local observer in $\mathrm{AdS}_{5}$, in the same way as the infinitesimal string mass $\mathrm{d} \bar{m}$ introduced above. Indeed, starting with the action for a point particle moving in the $\left(x^{1} \equiv x, z\right)$ plane,

$$
I_{P}=-m \int \mathrm{d} \tau \sqrt{-g_{00}\left(\frac{\partial x^{0}}{\partial \tau}\right)^{2}-g_{x x}\left(\frac{\partial x}{\partial \tau}\right)^{2}-g_{z z}\left(\frac{\partial z}{\partial \tau}\right)^{2}} \equiv \int \mathrm{d} \tau \mathcal{L},
$$

one can easily compute the canonical energy and momenta according to their definitions,

$$
P_{0}=\frac{\partial \mathcal{L}}{\partial \dot{x}^{0}}, \quad P_{x}=\frac{\partial \mathcal{L}}{\partial \dot{x}}, \quad P_{z}=\frac{\partial \mathcal{L}}{\partial \dot{z}},
$$

and thus check that the following representation holds for the mass of the particle,

$$
\sqrt{-g^{m n} P_{m} P_{n}}=\frac{z}{R} \sqrt{P_{0}^{2}-P_{x}^{2}-P_{z}^{2}}=m .
$$

Eq. (2.7) provides a natural generalization of this formula to the case of a string.

In view of the above, we conjecture that the string scale parameter $\gamma$ should have a similar physical interpretation: for a semiclassical string with large spin, or $R$-charge, the quantity $\gamma$ should be proportional to the anomalous dimension $\delta$ of the dual gauge theory operator. In the next section, we shall test this conjecture by comparing with known results for single-trace operators in $\mathcal{N}=4 \mathrm{SYM}$, as represented by rotating closed strings.

\section{$3 \quad$ Testing our conjecture versus closed strings}

In this section, we shall successively consider four different problems involving closed strings with large angular momenta, which have been studied at length in the literature, and for which the associated anomalous dimensions have been computed via the method introduced in ref. [9] — that is, by exploiting conformal symmetry. For all these strings, we shall compute the scale parameter $\gamma$ introduced in the previous section and verify that it is indeed proportional to the respective anomalous dimensions. 


\subsection{Closed string with high spin}

For a large spin $S$, the anomalous dimension of the twist-two operators made of adjoint fields, such as $\operatorname{Tr}\left(\phi^{I} D_{\left(\mu_{1} \ldots\right.} D_{\left.\mu_{S}\right)} \phi^{I}\right)$, is known to have the form [22]

$$
\Delta-S=f(\lambda) \ln (S)+\mathcal{O}\left(S^{0}\right),
$$

where $f(\lambda)$ is the cusp anomaly [3,4]. In the strong coupling limit of $\mathcal{N}=4$ SYM theory, such an operator is dual to a closed string rotating in global $\mathrm{AdS}_{5}$ space [9], with metric

$$
\mathrm{d} s^{2}=R^{2}\left(-\mathrm{d} \tau^{2} \cosh ^{2} \rho+\mathrm{d} \rho^{2}+\sinh ^{2} \rho \mathrm{d} \Omega_{3}^{2}\right) .
$$

The string is folded and stretched thus forming a very thin loop, made of two parallel straight lines, which rotates uniformly in the equator plane $(\rho, \phi)$ of $S^{3}: \phi=\omega \tau$. It extends up to a maximum value of the radial coordinate $\rho=\rho_{0}$, which is related to the angular velocity via

$$
\operatorname{coth}^{2} \rho_{0}=\omega^{2}
$$

It is understood that $\omega>1$. Note that all the coordinates and also the angular velocity are dimensionless, and that the physical dimensions are given by appropriate powers of the AdS radius $R$. Clearly, the string proper length is $\ell=4 R \rho_{0}$, where the factor of 4 comes by adding the four segments of the folded string. This, together with eq. (2.4), implies

$$
\gamma=4 R \int_{0}^{\rho_{0}} \mathrm{~d} \rho \sqrt{-g^{m n} \pi_{m}^{0} \pi_{n}^{0}}=\frac{2 R^{2}}{\pi \alpha^{\prime}} \rho_{0} .
$$

As shown by the first equality above, the same value for $\gamma$ can be inferred from the string energy-momentum densities that can be read off eqs. (3.6)-(3.7) below.

The Nambu-Goto Lagrangian has the form

$$
L=-\frac{4 R^{2}}{2 \pi \alpha^{\prime}} \int_{0}^{\rho_{0}} \mathrm{~d} \rho \sqrt{\cosh ^{2} \rho-\omega^{2} \sinh ^{2} \rho}
$$

from which the string energy and spin easily follow as (recall eq. (2.9))

$$
E=-4 \int_{0}^{\rho_{0}} \mathrm{~d} \rho \pi_{0}^{0}=\frac{4 R^{2}}{2 \pi \alpha^{\prime}} \int_{0}^{\rho_{0}} \mathrm{~d} \rho \frac{\cosh ^{2} \rho}{\sqrt{\cosh ^{2} \rho-\omega^{2} \sinh ^{2} \rho}},
$$

and respectively

$$
S=4 \int_{0}^{\rho_{0}} \mathrm{~d} \rho \pi_{\phi}^{0}=\frac{4 R^{2}}{2 \pi \alpha^{\prime}} \int_{0}^{\rho_{0}} \mathrm{~d} \rho \frac{\omega \sinh ^{2} \rho}{\sqrt{\cosh ^{2} \rho-\omega^{2} \sinh ^{2} \rho}} .
$$

The anomalous dimension is then obtained as $\delta=E-S$. (We recall that the energy $E$ in global $\mathrm{AdS}_{5}$ coincides with the total dimension $\Delta$ of the dual operator, and that $\delta=\Delta-(S+2) \simeq \Delta-S$ for twist-two operators with large spin $S \gg 1$.) 
There are two interesting limits (we recall that $R^{2} / \alpha^{\prime}=\sqrt{\lambda}$ ):

I. Large string with high spin $S \gg \sqrt{\lambda}$. This corresponds to the limit $\omega \rightarrow 1$. Writing $\omega=1+2 \eta$, with $\eta \ll 1$, we deduce from eq. (3.3) that $\rho_{0}$ is large,

$$
\rho_{0}=\frac{1}{2} \ln \left(\frac{1}{\eta}\right)+\mathcal{O}(1)
$$

and from eqs. (3.6)-(3.7) that the energy and spin are large as well [9]:

$$
E=\frac{\sqrt{\lambda}}{2 \pi}\left[\frac{1}{\eta}+\ln \left(\frac{1}{\eta}\right)+\ldots\right], \quad S=\frac{\sqrt{\lambda}}{2 \pi}\left[\frac{1}{\eta}-\ln \left(\frac{1}{\eta}\right)+\ldots\right]
$$

By using these equations, the scale factor in eq. (3.4) can be cast into the form

$$
\gamma \simeq \frac{\sqrt{\lambda}}{\pi} \ln \left(\frac{S}{\sqrt{\lambda}}\right)
$$

This is identical with the respective result for $\delta=E-S$, as emerging from eq. (3.9) [9]. Note that the way how this matching works is quite non-trivial: the dominant terms, of $\mathcal{O}(1 / \eta)$, in the energy $E$ and the spin $S$ precisely cancel in their difference, so the anomalous dimension $\delta$ is sensitive to the subleading terms of $\mathcal{O}(\ln (1 / \eta))$. By contrast, the scale parameter $\gamma$ is only logarithmically divergent as $\eta \rightarrow 0$ and hence, to the accuracy of interest, it is sensitive to the leading term in $S$ alone. What truly matters for our present purposes is that $\gamma$ and $\delta$ are proportional to each other, and hence have the same parametric dependencies upon $\sqrt{\lambda}$ and $S$. The fact that the proportionality coefficient appears to be exactly one is likely to be a coincidence (this will not happen for other examples below). Technically, this happens because, when $\omega \rightarrow 1$, the integrand in eqs. (3.6)-(3.7) for the difference $E-S$ approaches unity, and then the ensuing integral over $\rho$ is exactly the string length.

II. Small string with relatively low spin $S \ll \sqrt{\lambda}$. This corresponds to the limit of a large angular velocity $\omega \gg 1$. Then eq. (3.3) implies $\rho_{0} \simeq 1 / \omega$, while eqs. (3.6)-(3.7) yield [9]

$$
E \simeq \frac{\sqrt{\lambda}}{\omega}, \quad S=\frac{\sqrt{\lambda}}{2 \omega^{2}} \ll E,
$$

and therefore $\gamma \simeq(2 / \pi)(\sqrt{\lambda} / \omega)$ is again proportional with $\delta \simeq E \simeq \sqrt{\lambda} / \omega$, but the proportionality factor is now different from unity. In physical units, one has $\gamma \sim \delta \sim$ $\lambda^{1 / 4} \sqrt{S}$. Notice that this anomalous dimension is still large, $\delta \gtrsim \lambda^{1 / 4}$, albeit not as large as in the case of a high spin, cf. eq. (3.10).

\subsection{Closed string with large R-charge}

As another example, which has also been treated in ref. [9], let us consider a closed string rotating in $S^{5}$ with a large angular momentum $J$ and which is dual to a CFT operator with a large R-charge, equal to $J$. Choosing the metric on $S^{5}$ as

$$
R^{2} \mathrm{~d} \Omega_{5}^{2}=R^{2}\left(\mathrm{~d} \theta^{2}+\sin ^{2} \theta \mathrm{d} \phi^{2}+\cos ^{2} \theta \mathrm{d} \Omega_{3}^{2}\right),
$$


the string is folded, it rotates uniformly in the $(\theta, \phi)$-plane with $\phi=\omega t$, and it is stretched along the $\theta$ direction up to a value $\theta_{0}$ given by

$$
\sin \theta_{0}=\frac{1}{\omega}
$$

The proper length is $\ell=4 \int_{0}^{\theta_{0}} \sqrt{g_{\theta \theta}} \mathrm{d} \theta=4 R \theta_{0}$, where the factor of 4 comes from the four pieces of the folded string. The corresponding expressions for the string energy $E$, angular momentum $J$, and anomalous dimension $\delta=E-J$, can be found in ref. [9]. As before, there are two interesting regimes, (i) $\omega \rightarrow 1$ and (ii) $\omega \gg 1$, and in both cases the scale parameter $\gamma=\left(R / 2 \pi \alpha^{\prime}\right) \ell$ turns out to be proportional to $\delta$. Specifically, in the limit of very large R-charge $J \gg \sqrt{\lambda}$, corresponding to $\omega \rightarrow 1$, one finds $\theta_{0} \rightarrow \pi / 2$ and hence

$$
\gamma \simeq \frac{R^{2}}{\alpha^{\prime}}=\sqrt{\lambda}
$$

This differs by a factor $\pi / 2$ from the corresponding result for $\delta=E-J$ [9]. Once again, this agreement at parametric level is quite non-trivial: separately, $E$ and $J$ involve leadingorder contributions which diverge logarithmically in the limit $\omega \rightarrow 1$, but which mutually cancel in their difference. In the opposite limit where $\omega \gg 1$ and $J \ll E \ll \sqrt{\lambda}$, one finds $\theta_{0} \simeq 1 / \omega$, hence $\gamma \simeq(2 / \pi)(\sqrt{\lambda} / \omega)$, which differs by a factor $2 / \pi$ from the correct anomalous dimension in this regime, namely $\delta \simeq E \simeq \sqrt{\lambda} / \omega[9]$.

\subsection{Multi-spin closed string}

Consider now a multi-spin closed string, with two angular momenta $S_{1}$ and $S_{2}$ corresponding to rotations in two different directions of $\mathrm{AdS}_{5}$, as studied in detail in ref. [20]. We focus on the string solutions describing a circular closed string located at a fixed value of the $\mathrm{AdS}_{5}$ radius, while rotating simultaneously in two planes in $\mathrm{AdS}_{5}$ with equal spins $S_{1}=S_{2} \equiv S$. (This solution is a direct generalization of a two-spin flat-space solution where the string rotates in two orthogonal planes while always lying on a 3 -sphere.) Since the $\mathrm{AdS}_{5}$ radius is fixed, $\rho=\rho_{0}$, it suffices to consider the $S^{3}$ sector of the global metric in eq. (3.2), that is

$$
\mathrm{d} \Omega_{3}^{2}=\mathrm{d} \theta^{2}+\sin ^{2} \theta \mathrm{d} \phi^{2}+\cos ^{2} \theta \mathrm{d} \varphi^{2} .
$$

The string is rotating in the $\phi$ and $\varphi$ angles and winding once around the circle $0 \leq \theta<2 \pi$. So, the string length is $\ell=\int_{0}^{2 \pi} \sqrt{g_{\theta \theta}} \mathrm{d} \theta=2 \pi R \sinh \rho_{0}$. As announced, the spin components corresponding to rotations in the two angles have both the same magnitude,

$$
S_{1}=S_{2}=\frac{R^{2}}{\sqrt{2} \alpha^{\prime}} \sinh ^{3} \rho_{0}
$$

so the total spin strength is $S=\left(R^{2} / \alpha^{\prime}\right) \sinh ^{3} \rho_{0}$. The scale parameter $\gamma$ can be written as

$$
\gamma=\frac{R}{2 \pi \alpha^{\prime}} \ell=\frac{R^{2}}{\alpha^{\prime}} \sinh \rho_{0}=\frac{R^{2}}{\alpha^{\prime}}\left(\frac{S}{R^{2} / \alpha^{\prime}}\right)^{1 / 3},
$$

which has the same parametric form as the respective anomalous dimension computed in ref. [20], but is larger than the latter by a factor of $2 \sqrt{2} / 3$. 


\subsection{Giant magnon}

As an additional example of a closed string solution with a large angular momentum in the ten dimensional $\mathrm{AdS}_{5} \times \mathrm{S}^{5}$ space, let us now consider a giant magnon. This solution, discussed in detail in ref. [21], has an infinite angular momentum in the $\mathrm{S}^{5}$ space, corresponding to an infinite R-charge in the dual gauge theory. The $\mathrm{S}^{5}$ metric can be written in the form of eq. (3.12). For the solution possesing the least energy, one can use the results of ref. [21], and consider a fixed time string profile. From this solution at constant time, one finds the following string length:

$$
\ell=2 \int_{\theta_{0}}^{\pi / 2} \sqrt{g_{\theta \theta} \mathrm{d} \theta^{2}+g_{\phi \phi} \mathrm{d} \phi^{2}}=2 R \int_{\theta_{0}}^{\pi / 2} \frac{\sin \theta \cos \theta_{0}}{\sqrt{\sin ^{2} \theta-\sin ^{2} \theta_{0}}} \mathrm{~d} \theta,
$$

where the coordinate $\theta$ varies from $\pi / 2$ to $\theta_{0}$ and back to $\pi / 2$, and we used the relation between the angles $\phi$ and $\theta$ for fixed time. Performing this integral, one finds $\ell=R \pi \cos \theta_{0}$ and therefore $\gamma=R^{2} \cos \theta_{0} / 2 \alpha^{\prime}=\sqrt{\lambda} \cos \theta_{0} / 2$. This result for the scale factor $\gamma$ is equal to the anomalous dimension found in [21] multiplied by a factor of $\pi / 2$.

\section{Open strings and high spin mesons}

In this section we shall consider open strings rotating in $\mathrm{AdS}_{5}$, associated with mesons.

The system we want to describe consists of open strings rotating in $\mathrm{AdS}_{5}$ with endpoints attached to a probe D7 brane, which are dual to mesons in the $\mathcal{N}=2$ Yang Mills theory having a very large space-time spin $S$. As stated in the introduction, we expect these dual mesons to correspond to the leading-twist operators in the fundamental representation, as shown in eq. (1.2). More arguments supporting these interpretation will be provided by our results below.

In order to describe our string configuration, we work with $\mathrm{AdS}_{5}$ space in Poincaré coordinates and choose the metric as

$$
\mathrm{d} s^{2}=\frac{R^{2}}{z^{2}}\left(-\mathrm{d} t^{2}+\mathrm{d} z^{2}+\mathrm{d} r^{2}+r^{2} \mathrm{~d} \theta^{2}+\mathrm{d} y^{2}\right),
$$

where the spatial coordinates on the Minkowsky boundary are in cylindrical form: $(r, \theta, y)$. A high-spin meson is represented by a semiclassical rotating string with a steady profile $(r=r(z), \theta=\omega t, y=0)$, which is symmetric around $r=0$ and reaches a maximum value $z=z_{0}$ in the 'radial' direction [17]. The string endpoints are attached to a D7-brane located at $z=z_{D 7}$. The quark mass is related to the radial location of the brane via

$$
M_{q}=\frac{R^{2}}{2 \pi \alpha^{\prime} z_{D 7}} .
$$

As discussed in [17], it is convenient to use the dimensionless coordinates $\tilde{r} \equiv \omega r, \tilde{z} \equiv \omega z$. Chosing $\tau=t$ and $\sigma=\tilde{z}$, the Nambu-Goto action takes the form

$$
I=-\frac{R^{2} \omega}{\pi \alpha^{\prime}} \int \mathrm{d} t \int_{\tilde{z}_{D 7}}^{\tilde{z}_{0}} \mathrm{~d} \tilde{z} \frac{1}{\tilde{z}^{2}} \sqrt{\left(\tilde{r}^{\prime 2}+1\right)\left(1-\tilde{r}^{2}\right)}
$$


where a prime denotes a derivative with respect to $\tilde{z}$ and a factor of 2 has been included to account for the string symmetry around $r=0$. The string angular momentum is computed as (below $\left.\pi_{\theta}^{0}=\partial \mathcal{L} / \partial \omega\right)$

$$
S=2 \int_{z_{D 7}}^{z_{0}} \mathrm{~d} z \pi_{\theta}^{0}=\frac{R^{2}}{\pi \alpha^{\prime}} \int_{\tilde{z}_{D 7}}^{\tilde{z}_{0}} \mathrm{~d} \tilde{z} \frac{\tilde{r}^{2}}{\tilde{z}^{2}} \sqrt{\frac{\tilde{r}^{\prime 2}+1}{1-\tilde{r}^{2}}} .
$$

The string profile $\tilde{r}(\tilde{z})$ is determined by solving the following equation of motion:

$$
\frac{\tilde{r}^{\prime \prime}}{1+\tilde{r}^{\prime 2}}-\frac{2 \tilde{r}^{\prime}}{\tilde{z}}+\frac{\tilde{r}}{1-\tilde{r}^{2}}=0
$$

with a boundary condition stating that the string ends orthogonally on the D7-brane:

$$
\left.\frac{\mathrm{d} \tilde{r}}{\mathrm{~d} \tilde{z}}\right|_{\tilde{z}=\tilde{z}_{D 7}}=0
$$

and the following conditions at $\tilde{z}_{0}$, which express the symmetry of the string profile:

$$
\begin{gathered}
\tilde{r}\left(\tilde{z}_{0}\right)=0, \\
\left.\frac{\mathrm{d} \tilde{r}}{\mathrm{~d} \tilde{z}}\right|_{\tilde{z}=\tilde{z}_{0}} \rightarrow-\infty .
\end{gathered}
$$

Eqs. (4.5)-(4.8) determine the maximal string penetration $\tilde{z}_{0}$ as a function of the position $\tilde{z}_{D 7}$ of the D7-brane, and the string profile $\tilde{r}(\tilde{z})$ for $\tilde{z}_{D 7} \leq \tilde{z} \leq \tilde{z}_{0}$. In particular, the separation $L=2 \tilde{r}\left(\tilde{z}_{D 7}\right) / \omega$ between the string endpoints on the D7-brane is also interesting, in that it measures the typical size of the dual meson state in the gauge theory. We refer to ref. [17] for detailed numerical studies of the solutions and also for analytical approximations (some of which will be useful in what follows).

Calculating the string densities defined in eq. (2.6), one finds the scale parameter $\gamma$ as

$$
\gamma \equiv R \int_{\text {string }} \mathrm{d} \sigma \sqrt{-g^{m n} \pi_{m}^{0} \pi_{n}^{0}}=\frac{R^{2}}{\pi \alpha^{\prime}} \int_{\tilde{z}_{D 7}}^{\tilde{z}_{0}} \mathrm{~d} \tilde{z} \frac{1}{\tilde{z}} \sqrt{\tilde{r}^{\prime 2}+1} .
$$

From now on, we shall focus on operators with large spin $S \gg \sqrt{\lambda}$. As explained in ref. [17], this corresponds to the limit of a small angular velocity $\omega \rightarrow 0$; more precisely, the relevant, small, parameter is $\tilde{z}_{0} \ll 1$. This limit can be studied via a limited expansion in powers of $\tilde{z}_{0}$ around the well-known solution $\tilde{r}_{\text {st }}(\tilde{z})$ corresponding to a static string [23, 24]. In this regime, the integral in eq. (4.9) is proportional to the large logarithm $\ln \left(1 / \tilde{z}_{0}\right)$. Indeed, when $\tilde{z}_{0} \ll 1$, the lower limit $\tilde{z}_{D 7}$ is even smaller: one has $\tilde{z}_{D 7} \simeq C \tilde{z}_{0}^{3}$ with $C=$ 0.599 [17]. Also, to leading logarithmic accuracy, one can neglect the derivative $\tilde{r}^{\prime 2}$ under the square root, in spite of the fact that it diverges at the endpoint $\tilde{z}_{0}$, as required by eq. (4.8). To see this and also evaluate eq. (4.9) to the accuracy of interest, one can replace $\tilde{r}^{\prime}$ inside the integrand by the respective static solution [23, 24]:

$$
\tilde{r}_{\mathrm{st}}^{\prime}(\tilde{z})=\frac{\tilde{z}^{2}}{\sqrt{\tilde{z}_{0}^{4}-\tilde{z}^{4}}}
$$


(We have checked this approximation against the numerical calculation of the integral in eq. (4.9).) Then, one can successively rewrite the integral in eq. (2.5) as follows

$$
\begin{aligned}
\int_{\tilde{z}_{D 7}}^{\tilde{z}_{0}} \mathrm{~d} \tilde{z} \frac{1}{\tilde{z}} \sqrt{\tilde{r}_{\mathrm{st}}^{\prime 2}+1} & =\frac{1}{2} \int_{\varepsilon}^{1} \frac{d y}{y} \frac{1}{\sqrt{1-y^{2}}} \simeq \frac{1}{2} \int_{\varepsilon}^{1} \frac{d y}{y}+\frac{1}{2} \int_{0}^{1} \frac{d y}{y}\left(\frac{1}{\sqrt{1-y^{2}}}-1\right) \\
& =\ln \left(\frac{\tilde{z}_{0}}{\tilde{z}_{D 7}}\right)+D
\end{aligned}
$$

where $y \equiv\left(\tilde{z} / \tilde{z}_{0}\right)^{2}, \varepsilon \equiv\left(\tilde{z}_{D 7} / \tilde{z}_{0}\right)^{2}$, and in the last integral we were allowed to replace the lower limit by zero because $\varepsilon \ll 1$ and the integral is regular at that endpoint. Then this last integral is a pure number, evaluated as $D \simeq 0.347$. We thus obtain

$$
\gamma=\frac{2 \sqrt{\lambda}}{\pi}\left[\ln \left(\frac{1}{\tilde{z}_{0}}\right)+\mathcal{O}(1)\right],
$$

where we have also used $\tilde{z}_{D 7} \simeq C \tilde{z}_{0}^{3}$ and $\sqrt{\lambda}=R^{2} \alpha^{\prime}$. Still in this regime at $\tilde{z}_{0} \ll 1$, one can estimate the spin as [17]:

$$
S \simeq \frac{\sqrt{\lambda} C}{\pi \tilde{z}_{0}}
$$

which confirms that $S \gg \sqrt{\lambda}$, as anticipated. Let us also quote the respective result for the separation between the string endpoints on the D7-brane: $\tilde{r}\left(\tilde{z}_{D 7}\right) \simeq C \tilde{z}_{0}[17,23,24]$.

By eliminating $\tilde{z}_{0}$ between eqs. (4.12) and (4.13), one can express the scale parameter in terms of the spin $S$ :

$$
\gamma \simeq 2 \frac{\sqrt{\lambda}}{\pi} \ln \left(\frac{S}{\sqrt{\lambda}}\right) .
$$

This rewriting makes it clear that this parameter $\gamma$ is independent of the infrared scale in the gauge theory (the quark mass $M_{q}$ ), a necessary condition for the anomalous dimension of a local operator. It furthermore exhibits the right parametric dependencies upon $\lambda$ and $S$ (in particular, the characteristic $\ln S$-behavior) to be identified with the anomalous dimension of the quark-antiquark twist-two operator in eq. (1.2), up to a numerical coefficient. Hence the result in eq. (4.14) simultaneously supports our conjectured dualities between the rotating open string and the local operator (1.2) and, respectively, between the scale parameter $\gamma$ and the anomalous dimension $\delta$. As before, this last identification holds in the sense of a proportionality relation, in which the numerical coefficient is not under control. And indeed, the overall coefficient in eq. (4.14) differs by a factor of 4 from the correct respective value, as expected from the relation with the cusp anomaly for Wilson lines in the fundamental representation (cf. the discussion in the Introduction).

By inspection of eqs. (4.12)-(4.13), it is clear that the dimensionless quantity $\tilde{z}_{0}$ plays the same role within this open-string context as the (dimensionless) angular velocity $\omega$ within the closed-string calculation in section 3.1: this is a numerical AdS parameter which ( $i$ ) specifies the string theory object (here, an open string) which is dual to a gauge theory operator with a given spin, and (ii) parameterizes the relation between the spin and the anomalous dimension within the supergravity calculation. This parameter can be eventually eliminated between $S$ and $\gamma$, and then it totally disappears, as it should, from 
physical predictions such as eq. (4.14). From this perspective, $\tilde{z}_{0}$ should be viewed as a pure number in AdS, on the same footing as $m R$ in the case of an AdS particle (or a supergravity field) with mass $m$. There is however an interesting difference between these two dimensionless quantities, $m R$ and respectively $\tilde{z}_{0}=\omega z_{0}$, which reflects the peculiar way how conformal symmetry is broken by the introduction of the $\mathrm{D} 7$-brane in $\mathrm{AdS}_{5}$ and, related to that, the way how this symmetry is preserved in the calculation of special quantities, like the spin $S$ and the 'anomalous dimension' $\gamma$, which are guaranteed to be scale independent in the dual gauge theory. Namely, for a point particle in AdS, the parameter $m R$ is the product of the two scalars (in the sense of general coordinate transformations in $\mathrm{AdS}_{5}$ ): the 'particle mass' $m$ and the 'AdS radius' $R$. By contrast, in the problem of the rotating open string, neither $\omega$ nor $z_{0}$ are AdS scalars, rather they separately change under dilatations, but in such a way that their product remains invariant.

To see this, recall that an infinitesimal dilatation in the boundary gauge theory corresponds to an isometry of the bulk theory ${ }^{3}$ with the following $\mathrm{AdS}_{5}$ transformations:

$$
\begin{aligned}
z & \rightarrow(1+\epsilon) z ; & r & \rightarrow(1+\epsilon) r ; \\
\theta & \rightarrow \theta ; & \omega=\frac{\mathrm{d} \theta}{\mathrm{d} t} & \rightarrow \omega(1-\epsilon) .
\end{aligned}
$$

which leave the tilded variables $\tilde{r}$ and $\tilde{z}$ unchanged, This isometry changes the position $z_{D 7}$ of the D7-brane according to $z_{D 7} \rightarrow(1+\epsilon) z_{D 7}$, hence it is tantamount to a rescaling of the mass parameter $M_{q}$ in the dual gauge theory: $M_{q} \rightarrow(1-\epsilon) M_{q}$. However, quantities like $S$ and $\gamma$, which depend only upon the tilded variables $\tilde{z}_{D 7}$ or $\tilde{z}_{0}$, but not on $\omega$ or $z_{D 7}\left(=M_{q} R^{2} / 2 \pi \alpha^{\prime}\right)$ separately, are invariant under this transformation, meaning that they are independent of the mass scale $M_{q}$. More generally, the string equations of motion and their solutions are invariant under this isometry when written in terms of the tilded variables. (We implicitly used this symmetry to simplify the previous analysis.)

This situation should be contrasted with the string calculations of dimension-full quantities of the gauge theory, like the size $L$, or of the spectrum $E$, of the meson states. For a high spin meson, the size is obtained as $L \simeq 2 C \tilde{z}_{0} / \omega$. When this result is expressed in terms of the physical quantities $M_{q}$ and $S$, one finds $L \sim S^{2} /\left(\sqrt{\lambda} M_{q}\right)$, which represents a large size, $L \gg \sqrt{\lambda} / M_{q} \sim z_{D 7}$, when $S \gg \sqrt{\lambda}$. (Notice that $\Delta L \sim z_{D 7}$ is the quantum uncertainty in the size of the meson, as predicted by the UV/IR correspondence in AdS/CFT.) The meson energy is the same as the string energy measured by a gauge theory observer (cf. eq. (2.9)), that is [17]

$$
E=-2 \int_{z_{D 7}}^{z_{0}} \mathrm{~d} z \pi_{0}^{0}=\frac{R^{2} \omega}{\pi \alpha^{\prime}} \int_{\tilde{z}_{D 7}}^{\tilde{z}_{0}} \mathrm{~d} \tilde{z} \frac{1}{\tilde{z}^{2}} \sqrt{\frac{\tilde{r}^{\prime 2}+1}{1-\tilde{r}^{2}}} .
$$

Clearly, neither $L$ nor $E$ can be expressed as functions of the 'tilde' variables alone; rather, they also depend upon the mass scale $M_{q}$, as expected for bound states in a gauge theory.

One should perhaps emphasize here that the above arguments do not reduce to merely dimensional analysis, but rather involve subtle aspects of the AdS/CFT dictionary, like

\footnotetext{
${ }^{3}$ For the explicit form of the AdS isometries in Poincaré coordinates, see for example [25].
} 
the difference between quantities measured by gauge-theory observers as opposed to local observers in AdS. To see this, notice that if one was to evaluate the string energy as the sum of the energy densities measured by local observers, that is (cf. eq. (2.8))

$$
\bar{E} \equiv-2 \int_{z_{D 7}}^{z_{0}} \mathrm{~d} z \frac{z}{R} \pi_{0}^{0}=\frac{R}{\pi \alpha^{\prime}} \int_{\tilde{z}_{D 7}}^{\tilde{z}_{0}} \mathrm{~d} \tilde{z} \frac{1}{\tilde{z}} \sqrt{\frac{\tilde{r}^{\prime 2}+1}{1-\tilde{r}^{2}}}
$$

then the result $\bar{E}$ would be independent of the quark mass $M_{q}$, albeit it would still have mass dimension one, like the physical energy in eq. (4.16). We have no physical interpretation for the dimensionless quantity ${ }^{4} R \bar{E}$, but this is very similar to our string parameter $\gamma$, as it should be obvious from the discussion in section 2 .

To summarize, a local measurement in AdS yields results which are independent of the infrared scale and hence can be attributed to a local operator, whereas a coordinate-time observer measures properties of the gauge-theory state, which are also sensible to the mass scale $M_{q}$. So far, we have illustrated these two points of view using the examples of the scale parameter $\gamma$ ('local observer in AdS') and respectively the energy $E$ ('gauge theory observer'). It is interesting to similarly consider the case of the spin. Indeed, this is at the same time a property of the physical gauge state, and of the operator creating that state; hence the same value $S$ must be found both by a gauge theory observer, and by a local observer in AdS. This is indeed the case, as we now explain.

Recall first that, in the case of the energy, the difference between the string densities measured by the two types of observers is represented by the warp factor visible in eq. (2.8). Consider now the respective densities for the spin. The one seen by the gauge theory observer is $\mathrm{d} S / \mathrm{d} z=\pi_{\theta}^{0} \equiv \partial \mathcal{L} / \partial \omega$. As for the local observer, this measures a spin density

$$
\frac{\mathrm{d} \bar{S}}{\mathrm{~d} z}=\frac{\partial \overline{\mathcal{L}}}{\partial \bar{\omega}}
$$

where $\overline{\mathcal{L}}=(z / R) \mathcal{L}$ (this follows from $\mathrm{d} t \mathcal{L}=\mathrm{d} \bar{t} \overline{\mathcal{L}}$, with $\bar{t} \equiv \sqrt{g_{00}} t=(R / z) t$ ), while

$$
\bar{\omega}=\frac{\mathrm{d} \theta}{\mathrm{d} \bar{t}}=\frac{z}{R} \omega
$$

is the angular velocity measured by the local observer. Hence, eq. (4.18) implies $\mathrm{d} \bar{S} / \mathrm{d} z=$ $\mathrm{d} S / \mathrm{d} z$, and hence $\bar{S}=S$, as anticipated.

\section{Final comments}

Through our results in this paper, we brought strong indications in favor of two newly conjectured dualities, which support each other.

First, we have proposed a new recipe for computing anomalous dimensions at infinitely strong coupling for operators, from the $\mathcal{N}=4$ or $\mathcal{N}=2$ Yang Mills theories, which have large spin or large R-charge, and whose gravity duals are semiclassical strings rotating in the

\footnotetext{
${ }^{4}$ By itself, the 'energy' $\bar{E}$ cannot be a physical quantity in the gauge theory, since it is explicitly dependent upon the AdS scales $R$ and $\alpha^{\prime}$. However, when forming the dimensionless product $R \bar{E}$, these dependencies combine into the gauge-theory coupling factor $R^{2} \alpha^{\prime}=\sqrt{\lambda}$.
} 
$\mathrm{AdS}_{5} \times \mathrm{S}^{5}$ space-time (possibly supplemented by D7-branes). Specifically, we conjectured a proportionality relation between the anomalous dimensions of interest and the string scale factor $\gamma$ introduced in eq. (2.4), which is the string proper length in appropriate units. After testing our recipe against various problems involving closed strings, for which the anomalous dimensions can be independently and precisely computed via the method pioneered in ref. [9], we have considered the case of an open string with the endpoints attached to a D7-brane, for which the traditional method [9] fails to apply. For this problem, we found that the scale factor $\gamma$ has the same parametric behavior at large spin as the anomalous dimensions of twist-two operators, as known from closed-string calculations (for the single-trace operators built with adjoint fields), and also from the connexion to the cusp anomaly. This brought us to our second conjectured duality, that between a rotating open string with large spin and the quark-antiquark twist-two operator shown in eq. (1.2).

An interesting byproduct of our analysis is a distinction between two types of physical predictions from open string calculations in $\mathrm{AdS}_{5}$ with D7-branes. On one hand, there are observables related to string properties measured by a 'boundary' observer from the gauge theory; such observables express properties of the dual quantum state, like its energy or size, and depend upon the infrared scale in the gauge theory. On the other hand, there are quantities, like the scale parameter $\gamma$, which correspond to local measurements of the string by an observer living in ten dimensions; these quantities are independent of the mass scale in the gauge theory and are naturally interpreted as attributes of a local operator. A quantity like the spin enters both categories (a property of the operator and of the quantum state), and indeed it can be computed — on the string theory side — by either a local, or a boundary, observer, with identical results.

Admittedly, a fundamental understanding of these new dualities is still lacking. We have no rigorous justification for interpreting $\gamma$ as an anomalous dimension (for instance, we were not able to associate this quantity with the generator of some dilatation transformation), nor we understand the limitations of this interpretation (e.g., we do not know why the proportionality coefficient is not under control). Also, our current definition of $\gamma$ is restricted the temporal gauge and it is not clear whether this can be extended to a more general expression, which would make reparameterization invariance manifest. Also, we do not know whether it would make sense to introduce this parameter $\gamma$ for more general strings, undergoing some complicated motion (e.g. an infinite string with one endpoint attached to an accelerated heavy quark), and what should be its physical interpretation in that case. We hope that such questions will trigger further investigations, leading to conceptual clarifications and to new results.

For completeness, let us finally mention that anomalous dimensions corresponding to open string configurations with large angular momentum $J$, corresponding to large $R$-charge, have already been considered within the AdS/CFT correspondence, but for a different space-time geometry, which also involves a plane wave background. For example, ref. [26] discusses open strings in $\mathrm{AdS}_{5} \times \mathrm{S}^{5} / \mathrm{Z}_{2}$ with angular momentum $J$ in $S^{5}$ space. Both the anomalous dimension and the string length are found to be independent of $J$, which is consistent with our conjecture of a proportionality relation between these two quantities. In ref. [27], there is a discussion about open spinning strings with two $S^{5}$ 
spins in the same, plane wave, background. For these solutions, one can consider different limits of a large R-charge. We have tested the two particular cases where one keeps one of the spin components fixed, while letting the other component to approach infinity. The result is, again, that the anomalous dimension and the string length are independent of the R-charge, in agreement with the conjectured proportionality.

\section{Acknowledgments}

We would like to thank Steve Gubser, Juan Maldacena, Horatiu Nastase, and Carlos Nunez for enlightening correspondence, and Al Mueller and Ayan Mukhopadhyay for useful comments on the manuscript. This work was partially supported by the CAPES-COFECUB project $\mathrm{Ph} 663 / 10$. The work of E.I. is partially supported by the European Research Council under the Advanced Investigator Grant ERC-AD-267258. N.B. would like to thank the Institut de Physique Théorique de Saclay for warm hospitality on several occasions, and $\mathrm{CNPq}$ for partial support. E.I. would like to thank to whole group of the Theoretical Physics Department at UFRJ (Universidade Federal do Rio de Janeiro) for hospitality at various stages during the completion of this work.

Open Access. This article is distributed under the terms of the Creative Commons Attribution License (CC-BY 4.0), which permits any use, distribution and reproduction in any medium, provided the original author(s) and source are credited.

\section{References}

[1] D.J. Gross and F. Wilczek, Asymptotically free gauge theories. 2, Phys. Rev. D 9 (1974) 980 [INSPIRE].

[2] H. Georgi and H.D. Politzer, Electroproduction scaling in an asymptotically free theory of strong interactions, Phys. Rev. D 9 (1974) 416 [InSPIRE].

[3] G.P. Korchemsky, Asymptotics of the Altarelli-Parisi-Lipatov evolution kernels of parton distributions, Mod. Phys. Lett. A 4 (1989) 1257 [InSPIRE].

[4] G.P. Korchemsky and G. Marchesini, Structure function for large $x$ and renormalization of Wilson loop, Nucl. Phys. B 406 (1993) 225 [hep-ph/9210281] [INSPIRE].

[5] B. Basso and G.P. Korchemsky, Anomalous dimensions of high-spin operators beyond the leading order, Nucl. Phys. B 775 (2007) 1 [hep-th/0612247] [INSPIRE].

[6] J.M. Maldacena, The large- $N$ limit of superconformal field theories and supergravity, Adv. Theor. Math. Phys. 2 (1998) 231 [Int. J. Theor. Phys. 38 (1999) 1113] [hep-th/9711200] [INSPIRE].

[7] E. Witten, Anti-de Sitter space and holography, Adv. Theor. Math. Phys. 2 (1998) 253 [hep-th/9802150] [INSPIRE].

[8] S.S. Gubser, I.R. Klebanov and A.M. Polyakov, Gauge theory correlators from noncritical string theory, Phys. Lett. B 428 (1998) 105 [hep-th/9802109] [InSPIRE].

[9] S.S. Gubser, I.R. Klebanov and A.M. Polyakov, A Semiclassical limit of the gauge/string correspondence, Nucl. Phys. B 636 (2002) 99 [hep-th/0204051] [INSPIRE]. 
[10] N. Drukker, D.J. Gross and H. Ooguri, Wilson loops and minimal surfaces, Phys. Rev. D 60 (1999) 125006 [hep-th/9904191] [InSPIRE].

[11] M. Kruczenski, A note on twist two operators in $N=4 S Y M$ and Wilson loops in Minkowski signature, JHEP 12 (2002) 024 [hep-th/0210115] [INSPIRE].

[12] Y. Makeenko, Light cone Wilson loops and the string/gauge correspondence, JHEP 01 (2003) 007 [hep-th/0210256] [INSPIRE].

[13] A.V. Belitsky, A.S. Gorsky and G.P. Korchemsky, Gauge/string duality for QCD conformal operators, Nucl. Phys. B 667 (2003) 3 [hep-th/0304028] [INSPIRE].

[14] M. Kruczenski, R. Roiban, A. Tirziu and A.A. Tseytlin, Strong-coupling expansion of cusp anomaly and gluon amplitudes from quantum open strings in $A d S_{5} \times S^{5}$, Nucl. Phys. B 791 (2008) 93 [arXiv:0707.4254] [INSPIRE].

[15] L.F. Alday and J.M. Maldacena, Comments on operators with large spin, JHEP 11 (2007) 019 [arXiv:0708.0672] [INSPIRE].

[16] A. Karch and E. Katz, Adding flavor to AdS/CFT, JHEP 06 (2002) 043 [hep-th/0205236] [INSPIRE].

[17] M. Kruczenski, D. Mateos, R.C. Myers and D.J. Winters, Meson spectroscopy in AdS/CFT with flavor, JHEP 07 (2003) 049 [hep-th/0304032] [INSPIRE].

[18] J. Erdmenger, N. Evans, I. Kirsch and E. Threlfall, Mesons in gauge/gravity duals - A review, Eur. Phys. J. A 35 (2008) 81 [arXiv:0711.4467] [InSPIRE].

[19] R.A. Janik, P. Surowka and A. Wereszczynski, On correlation functions of operators dual to classical spinning string states, JHEP 05 (2010) 030 [arXiv:1002.4613] [INSPIRE].

[20] S. Frolov and A.A. Tseytlin, Multispin string solutions in $A d S_{5} \times S^{5}$, Nucl. Phys. B 668 (2003) 77 [hep-th/0304255] [INSPIRE].

[21] D.M. Hofman and J.M. Maldacena, Giant magnons, J. Phys. A 39 (2006) 13095 [hep-th/0604135] [INSPIRE].

[22] M.K. Benna, S. Benvenuti, I.R. Klebanov and A. Scardicchio, A test of the AdS/CFT correspondence using high-spin operators, Phys. Rev. Lett. 98 (2007) 131603 [hep-th/0611135] [INSPIRE].

[23] J.M. Maldacena, Wilson loops in large-N field theories, Phys. Rev. Lett. 80 (1998) 4859 [hep-th/9803002] [INSPIRE].

[24] S.-J. Rey and J.-T. Yee, Macroscopic strings as heavy quarks in large- $N$ gauge theory and Anti-de Sitter supergravity, Eur. Phys. J. C 22 (2001) 379 [hep-th/9803001] [INSPIRE].

[25] H. Boschi-Filho and N.R.F. Braga, Isometries of a D3-brane space, Class. Quant. Grav. 21 (2004) 2427 [hep-th/0311012] [INSPIRE].

[26] D.E. Berenstein, E. Gava, J.M. Maldacena, K.S. Narain and H.S. Nastase, Open strings on plane waves and their Yang-Mills duals, hep-th/0203249 [INSPIRE].

[27] B. Stefański Jr., Open spinning strings, JHEP 03 (2004) 057 [hep-th/0312091] [INSPIRE]. 